\title{
The addition of rituximab to fludarabine and cyclophosphamide improves progression-free survival in patients with previously treated chronic lymphocytic leukemia.
}

\author{
Amit Mahipal \\ Thomas Jefferson University \\ Mark Weiss \\ Thomas Jefferson University
}

Follow this and additional works at: https://jdc.jefferson.edu/medoncfp

Part of the Medical Immunology Commons, and the Oncology Commons

Let us know how access to this document benefits you

\section{Recommended Citation}

Mahipal, Amit and Weiss, Mark, "The addition of rituximab to fludarabine and cyclophosphamide improves progression-free survival in patients with previously treated chronic lymphocytic leukemia." (2010). Department of Medical Oncology Faculty Papers. Paper 2.

https://jdc.jefferson.edu/medoncfp/2

This Article is brought to you for free and open access by the Jefferson Digital Commons. The Jefferson Digital Commons is a service of Thomas Jefferson University's Center for Teaching and Learning (CTL). The Commons is a showcase for Jefferson books and journals, peer-reviewed scholarly publications, unique historical collections from the University archives, and teaching tools. The Jefferson Digital Commons allows researchers and interested readers anywhere in the world to learn about and keep up to date with Jefferson scholarship. This article has been accepted for inclusion in Department of Medical Oncology Faculty Papers by an authorized administrator of the Jefferson Digital Commons. For more information, please contact: JeffersonDigitalCommons@jefferson.edu. 


\title{
As submitted to: \\ Current Oncology Reports
}

And later published as:

\section{The Addition of Rituximab to Fludarabine and Cyclophosphamide Improves Progression-free Survival in Patients with Previously Treated Chronic Lymphocytic Leukemia}

\author{
Volume 12, Issue 6, November 2010, Pages 352-354
}

\section{DOI: $10.1007 / \mathrm{s} 11912-010-0122-3$}

Amit Mahipal \& Mark Weiss

Robak T, Dmoszynska A, Solal-Celigny P, et al.: Rituximab plus fludarabine and cyclophosphamide prolongs progression-free survival compared with fludarabine and cyclophosphamide alone in previously treated chronic lymphocytic leukemia. J Clin Oncol 2010, 28:1756-1765.

Rating

-Of importance.

Introduction

Chronic lymphocytic leukemia (CLL) is the most common form of leukemia in the United States [1]. There has been considerable progress in understanding the biology and treatment of CLL in last 20 years. The introduction of purine analogs (pentostatin, fludarabine, and cladribine) dramatically increased the response rates compared to alkylating agents $[2,3]$. Combination chemoimmunotherapy has further improved the overall frequency of response to over $80 \%$ in treatment-naïve and over $60 \%$ in relapsed/ refractory disease, respectively [4-7].

The combination of fludarabine, cyclophosphamide, and rituximab (FCR) has been demonstrated to be one of the most effective regimens in younger patients with CLL in phase 2 trials. Tam et al. [4], reporting on 300 patients, noted an overall response frequency of $95 \%$ with $72 \%$ achieving complete response (CR) in patients treated with FCR as initial therapy for CLL. Other studies of FCR, reported by Lamanna et al. [7] and Foon et al. [8], revealed an overall response frequency of $100 \%$ and $89 \%$, 
respectively, with CR in $77 \%$ and $61 \%$ of patients, respectively. In the salvage setting, FCR achieved a CR in $25 \%$ and overall response in $73 \%$ of the patients [5]. However, none of the prior studies have directly compared the addition of rituximab to fludarabine and cyclophosphamide in previously treated patients with CLL. Robak and colleagues report on a phase 3 trial, conducted at 88 centers in 17 countries between July 2003 and August 2007, that randomized previously treated patients with CLL to FC or FCR.

Aims

The aim of the REACH (Rituximab in the Study of Relapsed Chronic Lymphocytic Leukemia) study was to compare chemoimmunotherapy (FCR) with chemotherapy alone (FC) in patients with previously treated CLL. The primary end point of the study was progression-free survival (PFS). Response was assessed according to the National Cancer Institute (NCI)Working Group 1996 criteria with the addition of radiographic studies. Laboratory analyses were designed to assess risk-stratification parameters (ZAP70 expression, immunoglobulin heavy-chain variable region $[\mathrm{IgVH}]$ mutational status, CD 38 expression, cytogenetics and fluorescence in situ hybridization [FISH]), along with assessment of minimal residual disease by polymerase chain reaction for $\mathrm{IgVH}$ mutation.

Methods

The patients eligible for this trial had prior treatment of their CLL with one prior therapy that may have included chlorambucil, fludarabine, or an alkylator-containing combination regimen. Patients treated with alkylator/nucleoside combination regimens, interferon, rituximab, other monoclonal antibodies or stem cell transplantation were excluded from the trial. Patients were required to have adequate hepatic, renal, and bone marrow function, with an Eastern Cooperative Oncology Group performance status $\leq 1$ and a life expectancy $>6$ months. Patients were excluded if they had other invasive malignancy in the last 2 years, other serious medical condition, HIV, hepatitis B or C, and any comorbid condition requiring systemic corticosteroids for more than 1 month. All patients provided written informed consent. Patients were randomly assigned 1:1 to $\mathrm{FC}$ or FCR. All patients received intravenous fludarabine $25 \mathrm{mg} / \mathrm{m} 2 /$ day and cyclophosphamide $250 \mathrm{mg} / \mathrm{m} 2 /$ day both for 3 days, repeated every 28 days for the total of six cycles. Patients assigned to the FCR arm received rituximab $375 \mathrm{mg} / \mathrm{m} 2$ on day 1 of the first cycle and $500 \mathrm{mg} / \mathrm{m} 2$ on day 1 of the subsequent cycles. If a patient developed clinically significant grade 3 or 4 toxicity, chemotherapy dose was reduced by $25 \%$ and delay of $\geq 1$ week was scheduled. In patients with creatinine clearance between 30 to 70 $\mathrm{mL} / \mathrm{min}$, fludarabine dose was reduced by $25 \%$. All patients received prophylaxis for Herpes zoster and Pneumocystis carinii pneumonia with acyclovir/valacyclovir and cotrimoxazole, respectively. Allopurinol or rasburicase was administered to all patients for tumor lysis prophylaxis. Hematopoietic growth factors were not required but were permitted.

Toxicities were graded according to the NCI Common Toxicity Criteria (version 2.0). Quality of life was assessed using the Functional Assessment of Cancer Therapy-General 
(FACT-G) questionnaire (version 4.0). Response was graded according to the NCI Working Group 1996 criteria with the addition of imaging tests. Polymerase chain reaction for IgVH was used to assess minimal residual disease (MRD). Risk stratification parameters were determined using immunophenotyping, cytogenetics, and FISH analysis.

Results

Five hundred and fifty-two patients were enrolled and randomly assigned, with 276 patients in each arm. Six patients did not receive assigned treatment (four in FC arm and two in FCR arm). The baseline characteristics in the two arms were similar. The median age of patients in the FC and FCR arm was 62 years (range 35-81 years) and 63 years (range 35-83 years), respectively. In the $\mathrm{FC}$ arm, the numbers of patients in Binet stage A, B, and C were 31 (11\%), 160 (58\%), and 85 (31\%), respectively. The corresponding numbers in the FCR arm were $24(9 \%), 166(60 \%)$, and $86(31 \%)$, respectively. IgVH mutation was present in 92 (35\%) and $100(39 \%)$ patients in the FC and FCR arm, respectively. Twenty-four (9\%) patients in the FC arm and $18(7 \%)$ patients in the FCR arm had chromosome 17p deletion.

One hundred and sixty-seven (61.4\%) patients in the FC arm and 181 (67.5\%) patients in the FCR arm completed all six cycles of study treatment. The median follow-up time was 25 months. The hazard ratios of PFS and overall survival (OS) were 0.65 (95\% $\mathrm{CI}=0.51-0.82)$ and $0.83(95 \% \mathrm{CI}=0.59-1.17)$ for the FCR arm compared with the FC arm. The median PFS was 30.6 months in the FCR arm and 20.6 months in the FC arm $(\mathrm{P}<0.001)$. Statistically significant improvements were observed in the FCR arm in overall response rate $(69.9 \%$ vs $58 \%, \mathrm{P}=0.0034), \mathrm{CR}$ rate $(24.3 \%$ vs $13 \%, \mathrm{P}<0.001)$, median duration of response (39.6 vs 27.7 months, $\mathrm{P}=0.0252$ ), and median time to new treatment (not reached vs 34.3 months, $\mathrm{P}=0.0024$ ). Subgroup analysis demonstrated consistent improvement in PFS across various subgroups including poor prognostic factors like deletion 11q, unmutated IgVH, and positive ZAP-70. No benefit of FCR over FC therapy was reported in patients more than 70 years of age. There was no statistically significant difference in OS in the two groups, with median OS not reached in the FCR arm and 52 months in the FC arm ( $\mathrm{P}=0.2874)$. The differences in quality-of-life scores were small and not statistically significant. More patients achieving CR and receiving FCR (16 of 37 patients, 43\%) were MRD negative in peripheral blood than patients receiving FC (10 of 32 patients, 31\%).

The rates of overall adverse events (AEs), grade 3 or 4 AEs, serious AEs, and fatal AEs were higher in the FCR arm. Grade 3 or 4 adverse events were reported in $219(80 \%)$ patients and 200 (74\%) patients in the FCR and FC arm, respectively. Thirty six (14\%) patients in the FCR arm and $26(10 \%)$ in the FC arm experienced fatal AEs. A higher proportion of patients required dose modification or interruption $(51 \%)$ due to $\mathrm{AE}$ in the FCR arm than the FC arm (39\%), but treatment discontinuation due to AE was similar in the two arms (26\% vs $25 \%$ ). The most common nonhematologic AEs were nausea, vomiting, fever, fatigue, and diarrhea. Febrile neutropenia was reported in $12 \%$ of the patients in both arms. 
Conclusions

The authors concluded that FCR is superior to FC in previously treated patients with CLL. In this study, they reported improvement in PFS and response rates but no statistically significant difference in OS. The addition of rituximab to FC chemotherapy was well tolerated with higher incidence of AEs. There was no increase in overall or severe infections or proportion of patients requiring treatment discontinuation due to toxicity.

Comments

In this study, Robak et al. reported the benefit of chemoimmunotherapy compared to chemotherapy alone in patients with previously treated CLL [9]. The response rates in this trial are consistent with prior phase 2 trials using the identical combination. This is the only randomized controlled phase 3 trial in patients with previously treated CLL to demonstrate the benefit of addition of rituximab to chemotherapy. Prior phase 2 trials have compared FCR to historic controls. In this trial, there was a trend toward survival benefit but no statistically significant difference in $\mathrm{OS}(\mathrm{P}=0.2874)$.

The median age in this trial was 62 years, with only $17 \%$ of patients $>70$ years of age. Recognizing that the majority of patients diagnosed with CLL are older than 70 years of age, it is clear that this age group was underrepresented in this study. Consistent with other studies the improvement in PFS was not seen in patients older than 70 years, indicating that alternate strategies are needed for older patients $[4,10]$.

In clinical practice in the United States, rituximab is routinely administered as a part of therapy for CLL in both the previously treated and untreated groups. This study provides robust data to support the clinical practice of chemoimmunotherapy in patients with relapsed CLL. More recently, Hallek et al. [11] reported in abstract form improved PFS and OS in treatment-naïve patients with CLL receiving FCR compared with FC. Currently, the evidence from several clinical trials suggests that chemoimmunotherapy is the most post-anti-leukemic therapy for CLL. The main limitation to its application is that overlapping toxicity of these agents makes it difficult to administer safely to older patients with CLL.

\section{References}

1. Dighiero G, Hamblin TJ: Chronic lymphocytic leukaemia. Lancet 2008, 371:10171029.

2. Weiss MA, Maslak PG, Jurcic JG, et al.: Pentostatin and cyclophosphamide: an effective new regimen in previously treated patients with chronic lymphocytic leukemia. J Clin Oncol 2003, 21:1278-1284. 
3. Eichhorst BF, Busch R, Hopfinger G, et al.: Fludarabine plus cyclophosphamide versus fludarabine alone in first-line therapy of younger patients with chronic lymphocytic leukemia. Blood 2006, 107:885-891.

4. Tam CS, O'Brien S, Wierda W, et al.: Long-term results of the fludarabine, cyclophosphamide, and rituximab regimen as initial therapy of chronic lymphocytic leukemia. Blood 2008, 112:975-980.

5. Wierda W, O'Brien S, Wen S, et al.: Chemoimmunotherapy with fludarabine, cyclophosphamide, and rituximab for relapsed and refractory chronic lymphocytic leukemia. J Clin Oncol 2005, 23:4070-4078.

6. Lamanna N, Kalaycio M, Maslak P, et al.: Pentostatin, cyclophosphamide, and rituximab is an active, well-tolerated regimen for patients with previously treated chronic lymphocytic leukemia. J Clin Oncol 2006, 24:1575-1581.

7. Lamanna N, Jurcic JG, Noy A, et al.: Sequential therapy with fludarabine, high-dose cyclophosphamide, and rituximab in previously untreated patients with chronic lymphocytic leukemia produces high-quality responses: molecular remissions predict for durable complete responses. J Clin Oncol 2009, 27:491-497.

8. Foon KA, Boyiadzis M, Land SR, et al.: Chemoimmunotherapy with low-dose fludarabine and cyclophosphamide and high dose rituximab in previously untreated patients with chronic lymphocytic leukemia. J Clin Oncol 2009, 27:498-503.

9. Robak T, Dmoszynska A, Solal-Celigny P, et al.: Rituximab plus fludarabine and cyclophosphamide prolongs progression-free survival compared with fludarabine and cyclophosphamide alone in previously treated chronic lymphocytic leukemia. J Clin Oncol 2010, 28:1756-1765.

10. Eichhorst BF, Busch R, Stilgenbauer S, et al.: First-line therapy with fludarabine compared with chlorambucil does not result in a major benefit for elderly patients with advanced chronic lymphocytic leukemia. Blood 2009, 114:3382-3391.

11. Hallek M, Fingerle-Rowson G, Fink AM, et al.: First-line treatment with fludarabine (F), cyclophosphamide (C), and rituximab (R) (FCR) improves overall survival (OS) in previously untreated patients (pts) with advanced chronic lymphocytic leukemia (CLL): results of a randomized phase III trial on behalf of an international group of investigators and the German CLL study group [abstract 535]. Blood (ASH Annual Meeting Abstracts) 2009, 114:Abstract 535. 